\title{
Rsp5 ubiquitin ligase modulates translation accuracy in yeast Saccharomyces cerevisiae
}

\author{
MARTA KWWAPISZ, ${ }^{1,2}$ PIOTR CHOŁBIŃSKI, ${ }^{1}$ ANITA K. HOPPER, ${ }^{3}$ JEAN-PIERRE ROUSSET, $^{2}$ and \\ TERESA ŻOŁĄDEK ${ }^{1}$ \\ ${ }^{1}$ Department of Genetics, Institute of Biochemistry and Biophysics, Polish Academy of Sciences (PAS) , 02-106 Warsaw, Poland \\ ${ }^{2}$ Laboratoire de Génétique Moléculaire de la Traduction, Institut de Génétique et Microbiologie, Centre National de la \\ Recherche Scientifique (CNRS) UMR 8621, 91405 Orsay Cedex, France \\ ${ }^{3}$ Department of Biochemistry and Molecular Biology, Pennsylvania State University College of Medicine, Hershey, \\ Pennsylvania 17033, USA
}

\begin{abstract}
Rsp5p is an essential yeast ubiquitin protein ligase that ubiquitinates multiple proteins involved in various processes. Recent studies indicate that ubiquitination also affects translation. Here, we show that the strain with the rsp5-13 mutation exhibits altered sensitivity to antibiotics and a slower rate of translation. Using a sensitive dual-gene reporter system, we demonstrate that stop codon readthrough efficiency is decreased in the rsp5-13 mutant, while both +1 and -1 frameshifting were unaffected. The effect of the rsp5-13 mutation on readthrough could be reversed by increased expression of ubiquitin and partially suppressed by overproduction of the elongation factor eEF1A. As assessed by fluorescence in situ hybridization, the rsp5-13 mutant cells accumulate tRNA nuclear pools, perhaps depleting tRNA from the cytoplasm. Nuclear accumulation of tRNA is observed only when rsp5-13 cells are grown in media with high amino acid content. This defect, also reversed by overproduction of the elongation factor eEF1A, may be the primary reason for altered translational decoding accuracy.
\end{abstract}

Keywords: yeast; Rsp5 ubiquitin ligase; translation; tRNA export

\section{INTRODUCTION}

Ubiquitination is a post-translational modification of proteins that rapidly and reversibly changes in response to environmental stimuli or to programmed changes in cell state. Ubiquitination results from a cascade of reactions involving numerous enzyme components enabling a high degree of specificity and flexibility. In the first step of the cascade, an ubiquitin-activating enzyme (E1) activates ubiquitin in an ATP-dependent manner. In the second step, ubiquitin is transferred to a cysteinyl group on one of the ubiquitin-conjugating enzymes (E2s). Finally, through the action of a ubiquitin-protein ligase (E3), an isopeptide bond links ubiquitin to a particular substrate. The E3 proteins generally play a dominant role in substrate recognition and binding (for reviews, see Hershko and Ciechanover 1998; Schwartz and Hochstrasser 2003).

Distinct variants of ubiquitin chains regulate various processes: polyubiquitination involving ubiquitin lysines

Reprint requests to: Teresa Żołądek, Department of Genetics, Institute of Biochemistry and Biophysics, PAS, Pawińskiego 5A, 02-106 Warsaw, Poland; e-mail: teresa@ibb.waw.pl; fax: 4822-6584636.

Article published online ahead of print. Article and publication date are at http://www.rnajournal.org/cgi/doi/10.1261/rna.2131605.
48 and 29 directs short lived, damaged, misfolded, or misassembled proteins to the $26 \mathrm{~S}$ proteasome for degradation (Hochstrasser 1996; Hershko and Ciechanover 1998). In contrast, polyubiquitination involving lysine 63 (K63) regulates post-replicative DNA repair, transcription, cell cycle transitions, and endocytosis of plasma membrane proteins; monoubiquitination affects endocytosis/lysosomal degradation, meiosis, and chromatin remodeling (for reviews, see Hicke 2001; Weissman 2001; Lindsten et al. 2002).

Recent results indicate that ubiquitination also affects translation. Spence and colleagues (2000) have shown that active ribosomes are multiubiquitinated and that the L28 protein, located in the peptidyltransferase center, is ubiquitinated by the K63 multiubiquitin chain when incorporated in the ribosome. Moreover, a mutant strain solely expressing UbK63R and unable to form these variant chains showed altered sensitivity to translational inhibitors and a reduced translational rate in vitro. Proteomic studies revealed that many other ribosomal proteins, of both the small and large subunits, as well as translation elongation factor eEF1A, are ubiquitinated (Hitchcock et al. 2003; Peng et al. 2003), indicating that the regulatory effect of ubiquitination on translation might be quite complex. 
Rsp5p is an essential HECT domain containing ubiquitin-protein ligase in yeast. In addition to the catalytic HECT domain, Rsp5p possesses a C2 domain responsible for binding $\mathrm{Ca}^{2+}$, lipids, and proteins, and three WW domains that mediate protein-protein interactions (Harvey and Kumar 1999). Rsp5p interacts with itself in a twohybrid system and, therefore, is likely to be a multimeric protein (Dunn and Hicke 2001a). Rsp5p is localized in uniformly distributed punctate complexes in cells and cofractionates with a nonnuclear, nonmitochondrial, organellar subcellular fraction (Gajewska et al. 2001).

Rsp5p forms mono- or multi-ubiquitin chains linked via K63 of ubiquitin. Rsp5p impacts a wide variety of physiological processes, including regulation of endocytosis and lysosomal degradation of plasma membrane permeases such as Fur4p (Galan et al. 1996), Gaplp (Springael et al. 1999), Tat2p (Beck et al. 1999), and receptors such as Ste2p and Ste3p (Dunn and Hicke 2001b). Rsp5p also ubiquitinates a component(s) of the endocytic machinery (Dunn and Hicke 2001a; Gajewska et al. 2001; Kamińska et al. 2002; Stamenova et al. 2004). Moreover, Rsp5p-dependent ubiquitination is involved in sorting of amino acid permeases at the Golgi apparatus (Helliwell et al. 2001) and in the multivesicular bodies (Katzmann et al. 2004; Morvan et al. 2004). Rsp5p plays less well-characterized roles in other processes, including transcription, mitochondrial inheritance, the mitochondrial-cytoplasmic distribution of proteins, minichromosome maintenance, response to anesthetics, regulation of cellular $\mathrm{pH}$, biosynthesis of unsaturated fatty acids, and nuclear export of RNA (Huibregtse et al. 1995; de la Fuente et al. 1997; Wang et al. 1999; Wolfe et al. 1999; Hoppe et al. 2000; Kamińska et al. 2000; Neumann et al. 2003; Rodriguez et al. 2003; Shcherbik et al. 2003, 2004; Gwizdek et al. 2005). The mechanisms by which Rsp5p affects these various processes remain to be elucidated.

We previously identified $r s p 5$ mutations in a genetic selection for alterations in nonsense suppression, which indicated that Rsp5p might affect translation (Żoładek et al. 1995). In the present study we assessed the role of Rsp5p in this process. We showed that the rsp5-13 mutation alters cell sensitivity to antibiotics that act on translation and that it also affects the fidelity of translation. Additional copies of UBI1 encoding ubiquitin reverse the effect of the $r s p 5-13$ mutation on translation. Moreover, we show that an additional copy of TEF2, encoding eEF1A, suppresses the $r s p 5-13$ growth defects and translational phenotypes. Defects in fidelity of translation could be explained, at least in part, by tRNA nuclear accumulation displayed by cells with the $r s p 5-13$ allele.

\section{RESULTS}

\section{The rsp5-13 mutation affects translation}

The rsp5-13 mutation results in G(707)D substitution in the catalytic domain of Rsp5p, which causes changes in nonsense suppression and temperature-sensitive growth (Żołądek et al. 1997). We constructed isogenic strains to analyze the effect of $r s p 5-13$ mutation on translation and growth on media containing translational antibiotics. The rsp5-13 mutant grows slower than wild type, with a doubling time of $2 \mathrm{~h}, 43 \mathrm{~min}$ versus $1 \mathrm{~h}, 39 \mathrm{~min}$ for the wild type at $30^{\circ} \mathrm{C}$ at the beginning of the exponential growth $\left(t_{0}\right.$ to $t_{345}$ ), although growth resumes at a normal rate during the late exponential phase and reaches the same plateau. Consistent with slower growth, $\left[{ }^{35} \mathrm{~S}\right]$-methionine incorporation into proteins in cells grown in YPD at $30^{\circ} \mathrm{C}$ shows that the rsp5-13 mutant exhibits about a $70 \%$ decrease in the rate of total protein synthesis (Fig. 1). Slower protein synthesis could be due to defects in any step of translation: initiation, elongation, or in the generation or stability of ribosomes. To distinguish between these, we analyzed polysome profiles for wild-type and $r s p 5-13$ cells. Lysates were obtained from the wild type and the rsp5-13 mutant strain grown at $30^{\circ} \mathrm{C}$, as well as from these strains grown at $30^{\circ} \mathrm{C}$ and subsequently transferred for $3.5 \mathrm{~h}$ to $37^{\circ} \mathrm{C}$ to partially deactivate mutant Rsp5p (data not shown). The polysomes from wild-type and rsp5-13 cells were quite similar, indicating that the synthesis of ribosomes and polysomes stability is not disturbed in the rsp5-13 mutant strain, and therefore may not be responsible for the slower and thermosensitive growth phenotypes.

\section{The rsp5-13 mutant strain exhibits altered sensitivity to translational inhibitors}

Analysis of sensitivity of mutants to translational drugs can uncover underlying defects in translation (Masurekar et al. 1981). Our studies of the rsp5-13 mutant revealed its high sensitivity to the transpeptidylation inhibitor, anisomycin

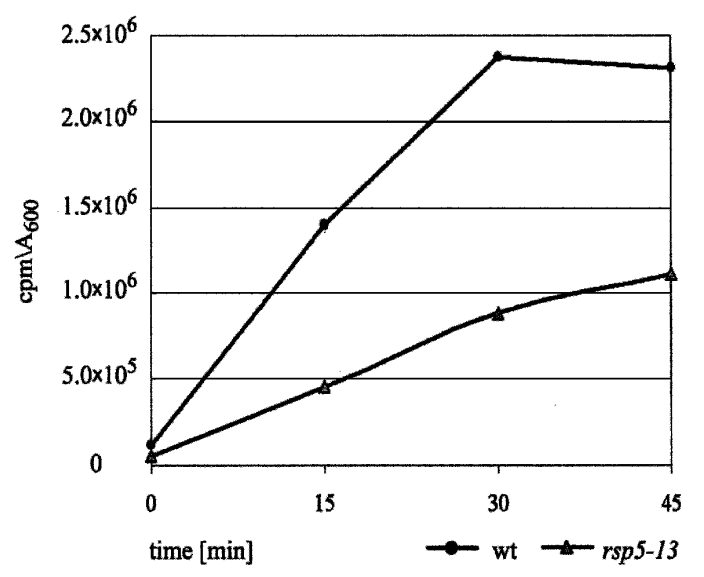

FIGURE 1. The rsp5-13 mutation affects the rate of protein synthesis. Wild-type cells (black circles) and $r s p 5-13$ mutant cells (triangles) were grown at $30^{\circ} \mathrm{C}$ to early exponential growth phase $\left(\mathrm{A}_{600}=0.5\right)$ in methionine-free synthetic medium, followed by $\left[{ }^{35} \mathrm{~S}\right]$-methionine labeling. Incorporation of radioactive methionine was measured at the indicated time points. 


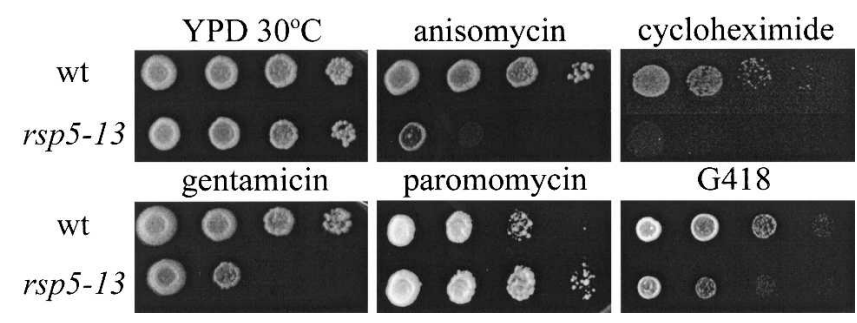

FIGURE 2. The rsp5-13 mutant exhibits altered sensitivity to translational drugs. Wild-type (MK1) and rsp5-13 (MK5) cells were grown to exponential phase. Serial 10-fold dilutions were spotted on YPD and media containing indicated antibiotics and incubated for 3 to $6 \mathrm{~d}$ at $30^{\circ} \mathrm{C}$.

(Fig. 2), which targets $25 \mathrm{~S}$ rRNA and the L3 protein (Rodriguez-Fonseca et al. 1995), and to cycloheximide (Fig. 2), an inhibitor of protein synthesis, which interferes with tRNA translocation when bound to the large subunit of the ribosome (Abraham and Pihl 1983). The rsp5-13 mutant was also resistant to paromomycin and moderately sensitive to the G418 and gentamicin (Fig. 2), aminoglycoside antibiotics that bind to the decoding region of the ribosomal A site and decrease decoding accuracy (Moazed and Noller 1987).

Sensitivity of the rsp5-13 strain to several translational antibiotics supports other data showing that Rsp5p affects translation. Addition of gentamicin, G418, or paromomycin causes lower fidelity of translation. Resistance to paromomycin usually correlates with the phenotype of higher fidelity of translation, antisuppression, or lower readthrough of stop codons, but there are exceptions to that rule (Dinman and Kinzy, 1997; Valente and Kinzy 2003). The different responses of the $r s p 5-13$ to these antibiotics indicate that the role of Rsp5p in translation may be complex.

\section{Readthrough of stop codons is reduced in the rsp5-13 strain and is restored by overexpression of $U B I 1$}

To test whether defects in Rsp5p-dependent ubiquitination influence the fidelity of translation, readthrough of stop codons and frameshifting were measured in the rsp5-13 mutant and in the wild-type strains using a dual-gene reporter system. This system consists of vectors containing readthrough or frameshift-promoting signals inserted between the lac $Z$ and luc sequences, encoding $\beta$-galactosidase and luciferase, respectively (Stahl et al. 1995). Luciferase activity reflects the readthrough or frameshifting efficiency, while $\beta$-galactosidase activity serves as a general control of expression level, integrating a number of possible sources of variability (plasmid copy number, transcriptional activity, mRNA stability, and translation rate). Our results showed that the Rsp5p defect has no effect on the frequency of -1 and +1 frameshift events (data not presented). However, the rsp5-13 mutant showed an almost twofold lower level of UAA ( $p$-value $=0.00001$ ), UGA ( $p$-value $=0.00217)$, and UAG $(p$-value $=0.0002)$ stop codon readthrough than the parental strain (Fig. 3A).
Maintenance of proper free ubiquitin levels is critical for cell viability, particularly when cells are challenged by stress or drug treatment (Hanna et al. 2003). Ubiquitin is encoded by four genes, $U B I 1, U B I 2$, and $U B I 3$, supplying the bulk ubiquitin in favorable growth conditions, and UBI4, expressed under stress conditions (Ozkaynak et al. 1984; Finley et al. 1987, 1989). These genes have been previously found to act as multicopy suppressors of the temperature-sensitive growth of $r s p 5$ mutants (Żołądek et al. 1997; Kabir et al. 2005). Here we checked whether suppression of growth is correlated with suppression of translational defects.

We monitored translational readthrough for rsp5-13 $[U B I 1]_{\mathrm{N}}$ and wild-type $[U B I 1]_{\mathrm{N}}$ transformants expressing ubiquitin from a multicopy plasmid. Only a small difference in readthrough between the wild type and the rsp5-13 mutant overexpressing ubiquitin was detected (Fig. 3A,B), indicating that an excess of ubiquitin produced from UBI1 gene partially suppresses the rsp5-13 effect on readthrough of stop codons. Neither overexpression of ubiquitin nor empty vector (not shown) has an effect on readthrough in the wild-type strain. An additional copy of the RSP5 gene or its overexpression from a multicopy plasmid had no effect on readthrough of the wild-type strain (data not shown). Our data support the notion that both Rsp5p ubiquitin ligase and an active ubiquitination pathway are necessary for the maintenance of normal fidelity of translation.

\section{rsp5-13 defect of readthrough is suppressed by an additional copy of TEF2}

The elongation factor eEF1A, which delivers tRNAs to the Asite of the ribosome, is encoded by two nearly identical genes, $T E F 1$ and TEF2, and mutations in these genes affect fidelity of
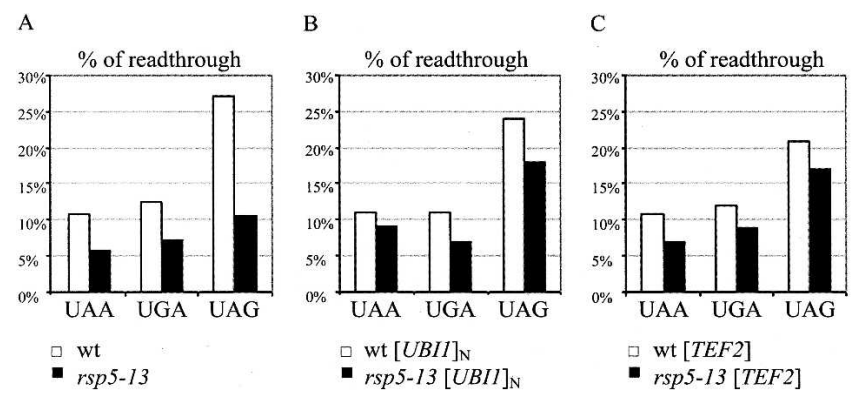

FIGURE 3. Effect of $r s p 5-13$ mutation and extra copies of UBI1 and TEF2 genes on stop codon readthrough. (A) rsp5-13 mutant shows a twofold lower level of stop codon readthrough than the parental strain. Measurements were made in wild-type (MK1) and $r s p 5-13$ (MK5) strains. Details are given in Materials and Methods. (B) Overexpression of $U B I 1$ gene suppresses the phenotype of low stop codon readthrough in the $r s p 5-13$ strain. Measurements were made in wild type (MK1) and rsp5-13 (MK5) transformed with YEp96 plasmid expressing ubiquitin. (C) Overexpression of TEF2 gene suppresses the phenotype of low stop codon readthrough in rsp5-13 strain. The level of readthrough was measured in wild type (MK1) and rsp5-13 (MK5) transformed with YCp-TEF2 plasmid. 
translation (Song et al. 1989; Dinman and Kinzy 1997; CarrSchmid et al. 1999). To investigate a possible functional interaction between eEF1A and Rsp5p, TEF2 was expressed from a centromere containing plasmid (maintenance in about 1 copy/cell) in wild-type and rsp5-13 mutant strains and the level of readthrough was measured. The difference between these strains was smaller than that between the wildtype and rsp5-13 strains lacking YCp-TEF2. This indicates that TEF2 functions as a partial suppressor of the readthrough defect in rsp5-13 cells (Fig. 3C). Expression of an additional copy of the TEF2 gene had no effect on readthrough in the wild-type strain (Fig. 3, cf. C and A). As anticipated from these results, an additional copy of the TEF2 gene also corrected the thermosensitive growth of rsp5-13 cells, since the rsp5-13 [TEF2] transformants grew on YPD and minimal medium at $34^{\circ} \mathrm{C}$ better than rsp5-13 transformed with the vector alone (Fig. 4). Moreover, the additional copy of the TEF2 gene enabled the rsp5-13 mutant to grow better in the presence of cycloheximide. However, there was little effect of TEF2 additional copy expression on growth on anisomycin and G418-containing medium (Fig. 4). The genetic interactions suggest that the rsp5-13 mutation may affect translation at the elongation step. However, we did not detect differences in polysome profiles in the rsp5-13 mutant compared to wild type, perhaps because polysome analysis is not sensitive enough to detect the changes uncovered by the drugs and readthrough assay.

\section{An additional copy of TEF2 suppresses tRNA nuclear accumulation caused by rsp5-13}

Readthrough of stop codons depends on ribosomal proteins, translation factors, and many proteins associated with the ribosome, such as chaperones and actin cytoskeleton (for reviews, see Kandl et al. 2002; Valente and Kinzy 2003). The distribution of tRNA in the cell, its quality, aminoacylation, and modifications, association with eEF1A, and/or accessibility to the translational apparatus also affect the fidelity of protein synthesis and, in particular, the termination step.

We analyzed the subcellular distribution of tRNA by fluorescence in situ hybridization (FISH). The rsp5-13 mutant cells, in contrast to the wild-type strain, accumulate large amounts of nuclear tRNAs. Nuclear accumulation of tRNA in rsp5-13 mutant cells was observed after $3.5 \mathrm{~h}$ of incubation at $37^{\circ} \mathrm{C}$ or $33.5^{\circ} \mathrm{C}$ using digoxigenin (DIG)labeled probes specific for $\mathrm{tRNA}^{\mathrm{Tyr}}$, encoded by introncontaining genes, or tRNA ${ }^{\mathrm{Met}}$, encoded by intronless genes (Fig. 5A and see 5C). These findings are in agreement with previous observations showing Rsp5p involvement in nuclear export of tRNA (Neumann et al. 2003). In agreement with other published data (Rodriguez et al. 2003), poly(A)-containing RNA was also found to accumulate in the nucleus of $r s p 5-13$ cells at the nonpermissive temperature (Fig. 5A).

We discovered a surprising effect of nutritional conditions upon tRNA subcellular distribution. Nuclear accumulation of both types of tRNAs was observed in rsp5-13 mutant cells grown in rich YPD or SD + casamino acids media, but nuclear tRNAs were not detected when these cells were grown to logarithmic phase in SC-ura medium, which contained all amino acids in concentrations described by Sherman (2002) (Fig. 5B). The second and last media differ only in concentrations of amino acids, indicating that these are components important for Rsp5p action in tRNA transport. These data indicate further that wild-type Rsp5 ligase activity is necessary for appropriate tRNA nucleus/cytosol distribution only when amino acids are abundant.

An additional copy of TEF2 suppresses the defect in the translational readthrough level provoked by $r s p 5-13$ mutation and improves thermosensitive growth of mutant strain; thus we checked whether the tRNA nuclear accumulation is ameliorated in rsp5-13 cells overexpressing the TEF2 gene. Wild type with vector alone [-], rsp5-13 [-], and rsp5-13 [TEF2] strains were pregrown in SD + casamino acids medium, grown in YPD at $23^{\circ} \mathrm{C}$ overnight, and shifted to $33.5^{\circ} \mathrm{C}$ for $3.5 \mathrm{~h}$. FISH was performed using a probe hybridizing to intron-containing pre-tRNA ${ }^{\text {Tyr }}$ and processed $\mathrm{tRNA}^{\mathrm{Tyr}}$. In contrast to $r s p 5-13[-]$, no nuclear accumulation of tRNA was observed in rsp5-13 [TEF2] (Fig. 5C). The data indicate that overproduced eEF1A suppresses the rsp5-13 defect in subcellular distribution of tRNA as well as translational readthrough and growth defects in the absence and presence of antibiotics.

\section{DISCUSSION}

Several, mostly unexplained, relationships between the ubiquitin system and protein synthesis have been previously

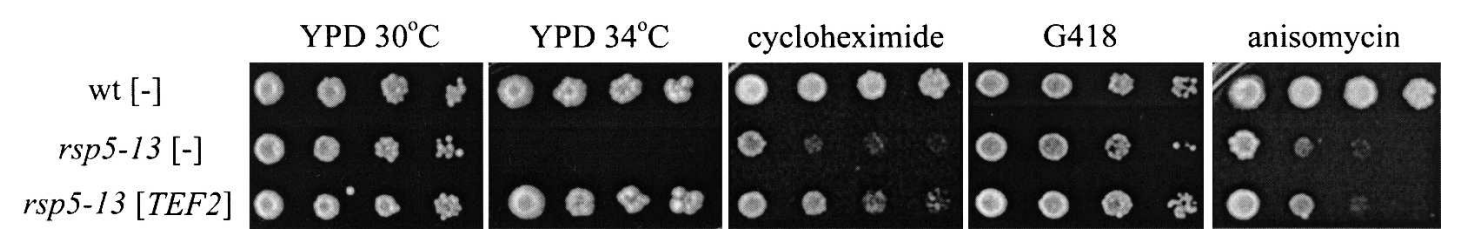

FIGURE 4. An additional copy of TEF2 gene improves growth of $r s p 5-13$ mutant at elevated temperature and on media containing translational antibiotics. Growth of wild-type and $r s p 5-13$ strains transformed with the vector and $r s p 5-13$ strain transformed with YCp-TEF2 was compared after plating serial dilutions of cultures on YPD or YPD containing indicated antibiotics and incubation for $3-6 \mathrm{~d}$ at $30^{\circ} \mathrm{C}$ or $34^{\circ} \mathrm{C}$. 
A

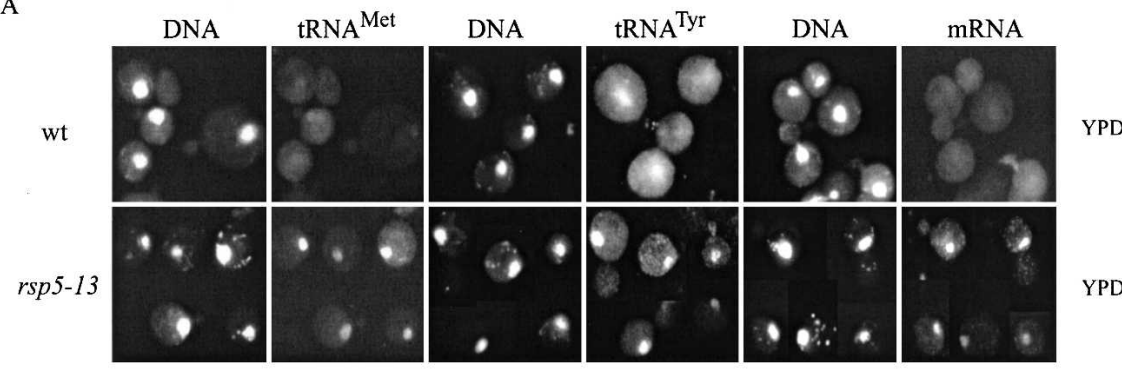

B

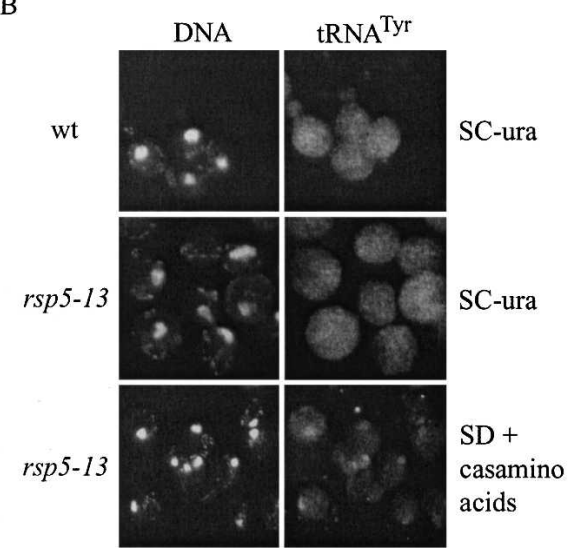

$\mathrm{C}$

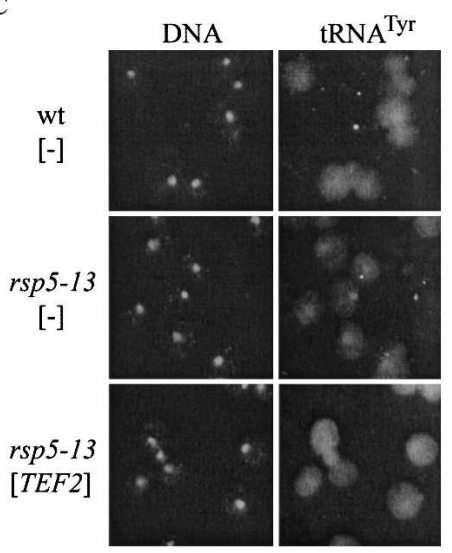

FIGURE 5. $r s p 5-13$ mutation causes accumulation of tRNA in the nucleus and an additional copy of TEF2 gene reverses this effect. (A) Wild-type and $r s p 5-13$ strains were grown at $23^{\circ} \mathrm{C}$ in YPD medium and shifted for $3.5 \mathrm{~h}$ to $37^{\circ} \mathrm{C}$. FISH was performed using specific digoxigeninlabeled probes recognizing intron-containing pre-tRNA and mature tRNA (tRNA ${ }^{\mathrm{Tyr}}$ ), intronless tRNA ${ }^{\text {Met }}$, and probes specific for polyA. DNA was stained with DAPI. (B) Wild-type and $r s p 5-13$ strains were grown at $23^{\circ} \mathrm{C}$ in SC-ura or SD + casamino acids medium and shifted for $3.5 \mathrm{~h}$ to $37^{\circ} \mathrm{C}$. FISH was performed using specific digoxigenin-labeled probes recognizing tRNA $^{\text {Tyr }}$. DNA was stained with DAPI. (C) Wild-type [vector], rsp5-13 [vector] and $r s p 5-13$ [TEF2] strains were grown at $23^{\circ} \mathrm{C}$ and shifted for $3.5 \mathrm{~h}$ to $33.5^{\circ} \mathrm{C}$. FISH was performed using specific digoxigenin-labeled probes recognizing tRNA ${ }^{\mathrm{Tyr}}$. DNA was stained with DAPI.

observed. In this report we show that a mutant deficient in Rsp5 ubiquitin-protein ligase is hypersensitive to drugs that inhibit translation and resistant to a drug that causes misreading. Mutant $r s p 5-13$ cells grow slower and exhibit a lower rate of translation compared to the parental strain. We also observed a significant decrease of translational readthrough in the rsp5-13 mutant, although both +1 and -1 frameshifting remained unaltered, suggesting that competition between natural suppressor tRNAs and release factors favors the latter. Furthermore, the rsp5-13 mutation also causes tRNA nuclear accumulation, which may account for its antisuppressor phenotype.

The rsp5-13 mutant cells exhibit a broad spectrum of sensitivity to translational inhibitors, but are particularly resistant to paromomycin, which strongly stabilizes the A-site binding of near-cognate peptidyl-tRNAs and increases misreading (Ogle et al. 2001; Vicens and Westhof 2001). This correlates with an almost twofold decrease of readthrough at all three stop codons in $r s p 5-13$ mutant cells compared to wild-type cells.
There is no effect of ubiquitin levels on nonsense suppression in the wild-type cells (Chernova et al. 2003; see Results). However, in the presence of the prion form of the translational termination complex component, Sup35p $\left[P S I^{+}\right]$, changes in the free ubiquitin pool affect suppression efficiency. Moreover, the ubiquitin pool also affects cellular responses to drugs that influence translation. For example, ubp $6 \Delta$ mutant cells, lacking the de-ubiquitinating enzyme, have a lowered ubiquitin pool and are hypersensitive to cycloheximide and anisomycin, drugs affecting translation (Keeven et al. 2002; Chernova et al. 2003; Hanna et al. 2003). Thus, $u b p 6 \Delta$ resembles the rsp5-13 mutant in this respect. However, the altered antibiotic sensitivity of ubiquitin pathway mutants may reflect effects on various pathways, not only translation. Recently, it was demonstrated that the pool of ubiquitinated proteins and free ubiquitin levels are highly reduced in the $r s p 5-1$ (L733S) mutant, especially at elevated temperature (Krsmanovic and Kolling 2004). Accordingly, the low readthrough levels in $r s p 5-13$ cells might be related to a diminished ubiquitin pool that appears to be about $75 \%$ of wild-type levels (M. Kwapisz, J.-P. Rousset, and T. Żołądek, unpubl. results), although the pool of ubiquitinated proteins is similar in $r s p 5$ 13 and in wild type (data not shown). Supporting the role of the ubiquitin pool is our data showing that the nonsense codon readthrough phenotype of $r s p 5-13$ cells is suppressed by overexpressed ubiquitin.

At least two mechanisms may account for the effect of free ubiquitin and the rsp5-13 mutation on stop codon readthrough. (1) Ubiquitination may directly affect an element of the translational apparatus functioning in the termination process. Indeed, several such factors have been found to be ubiquitinated (Hitchcock et al. 2003; Peng et al. 2003). However, we were unable to demonstrate ubiquitination of eRF3/Sup35p and the cellular level of this factor was similar in wild-type and rsp5-13 strains (our unpubl. observations). (2) Alternatively, and the model we favor, changes in other processes requiring ubiquitination may indirectly affect stop codon recognition.

There are previous reports documenting that translation rate and/or nonsense codon suppression can be affected indirectly via alterations in tRNA distribution, accessibility, and properties, by altered tRNA modification, and/or by factors regulating tRNA levels (Dihanich et al. 1987; Hurt 
et al. 1987; Beier and Grimm 2001; Kwapisz et al. 2002; Lecointe et al. 2002). For example, the yeast Los1p was first identified by its loss of nonsense suppression phenotype (Hopper et al. 1980). Los1p functions as a nuclear exportin for tRNA, providing one of two or more pathways to deliver tRNA from the nucleus to the cytosol (Hurt et al. 1987; Hellmuth et al. 1998; Sarkar and Hopper 1998; for reviews, see Grosshans et al. 2000b; Hopper and Phizycky 2003). Thus, the altered nucleus/cytosol distribution of tRNA evidenced by the rsp5-13 mutant cell (shown previously for $r s p 5-3$ by Neumann et al. 2003) might be the cause of its defects in translation efficiency and fidelity. If this is the case, then suppression of $r s p 5-13$ by additional eEF1A might result either from more efficient utilization of the residual cytoplasmic pool of tRNA or by a role of eEF1A in tRNA nuclear export, as has been previously proposed (Grosshans et al. 2000a). Neumann et al. (2003) reported also that $r s p 5$ mutants show defects in pre-rRNA processing, and this may possibly contribute to the changes in translation rate and fidelity we observe.

Why does mutation of RSP5 cause altered distribution of tRNA between the nucleus and the cytoplasm? Direct modification by Rsp5p of Los1p and/or other components of the machinery governing RNA nuclear transport could provide one such mechanism. Indeed, a genome-wide study identified Los1p as an ubiquitinated protein (Peng et al. 2003). Alternatively, Rsp5p may be involved in a nutrient-dependent signaling process that affects the distribution of tRNA between the nucleus and the cytoplasm. Retrograde tRNA movement of tRNA has been demonstrated (Shaheen and Hopper 2005; Takano et al. 2005) and has been shown to be responsive to nutrient deprivation (Shaheen and Hopper 2005). Moreover, as shown by Grosshans and colleagues (2000a), amino acid deprivation results in tRNA nuclear accumulation. Although Rsp5p alters cellular amino acid permease sorting (Hein et al. 1995; Galan et al. 1996; Gajewska et al. 2001), others have shown that such inappropriate permease sorting need not dramatically alter internal amino acids pools (Crespo et al. 2004). Thus, Rsp5p unlikely affects tRNA distribution via altered cellular amino acid pools. Since Rsp5p functions in regulation of the transcription factors, Spt23p, Mga2p, and Gln3p (Hoppe et al. 2000; Shcherbik et al. 2003; Crespo et al. 2004), tRNA nuclear accumulation in rsp5-13 cells, only when grown in media with high amino acids concentrations, could be rather a response to an altered transcriptional program or a result of direct involvement of Rsp5p in amino acid signaling.

During the cell life translation maintains a balance between speed and accuracy (Kurland 1992) and cell may gain in fitness if translational accuracy varies in response to growth conditions and nutrient accessibility. The translational accuracy is sensitive to tRNA accessibility and its distribution in the cell. The role of Rsp5p-dependent ubiquitination could be general and complex, and would facilitate the traffic of RNA and various proteins in the cell in the response to changing physiological conditions, especially amino acid accessibility. It is likely that several Rsp5p substrates are involved in the regulation of nuclear export systems, but that remains to be determined.

\section{MATERIALS AND METHODS}

\section{Strains, media, and genetic procedures}

Yeast strains used in this study are: MHY501 MAT $\alpha$ his3- $\Delta 200$ leu2-3,112 ura3-52 lys2-801 trp1-1 (Chen et al. 1993), MK1 (MHY501 but HA-RSP5; this work), and MK5 (MHY501 but HArsp5-13; this work). Escherichia coli strain $\mathrm{DH} 5 \alpha \mathrm{F}^{\prime}$ ( $F^{\prime}$ supE44 lacU169 [80 lacZM15] hsdR17 recA1 endA1 gyrA96 thi-1 relA1) was used for cloning and plasmid propagation. For replacement of the RSP5 gene, integration plasmids YIpHA-RSP5/rsp5-13 bearing the RSP5 or $r s p 5-13$ mutant version and the triple hemagglutinin (HA)-epitope tag (see below; Gajewska et al. 2001) were linearized with PstI (Promega) or AgeI (Promega) and transformed into the MHY501 strain. Integrants were selected on SC-ura plates and then incubated on $\mathrm{SD}+5^{\prime}$ fluorouracil $\left(5^{\prime} \mathrm{FOA}\right)$ plates (see below) to select for cells that had lost the URA3 marker. Allele replacement was confirmed by PCR, and Rsp5p HA-tagging was confirmed by Western blot using antibodies against the HA epitope (clone 12CA5, BabCo).

E. coli cells were grown in LB medium supplemented with appropriate antibiotics to maintain the plasmids. The following media were used for yeast growth: YPD ( $2 \%$ glucose, $1 \%$ peptone, $1 \%$ yeast extract), SD ( $2 \%$ glucose, $0.67 \%$ yeast nitrogen base without amino acids), SD + casamino acid (SD supplemented with $1 \%$ casamino acids and $20 \mathrm{mg} / \mathrm{L}$ tryptophan) used for FISH experiments, SD + CSM (SD supplemented with $0.57 \%$ complete synthetic media lacking leucine or lacking leucine and uracil or lacking leucine and tryptophan, from ICN) used for fidelity of translation measurements, and SC-ura (SD supplemented with all amino acids and adenine $40 \mathrm{mg} / \mathrm{L}$ but lacking uracil, according to Sherman 2002), used for FISH experiments and strain construction. Solid media contained $2 \%$ agar. The amounts of drugs used were as follows: $0.22 \% 5^{\prime} \mathrm{FOA}, 0.1 \mu \mathrm{g} / \mathrm{mL}$ cycloheximide, $1.0 \mathrm{mg} / \mathrm{mL}$ paromomycin, $10 \mu \mathrm{g} / \mathrm{mL}$ anisomycin, $50 \mu \mathrm{g} / \mathrm{mL}$ G418, $10 \mu \mathrm{g} / \mathrm{mL}$ gentamicin. Paromomycin- and gentamicin-containing plates were buffered with 100 $\mathrm{mM}$ potassium phosphate buffer ( $\mathrm{pH} 7.5-8.0)$. In all cases, growth of wild-type cells was not inhibited at the drug concentrations used.

Suppression of both the temperature-sensitive phenotype and antibiotics sensitivity was monitored by a drop test. An appropriate strain was suspended in water and serial 10-fold dilutions of cells were spotted onto solid media and incubated for 2-6 d at indicated temperatures. The growth rate in liquid cultures was monitored by $\mathrm{A}_{600}$ measurements. Standard yeast genetic methods were employed (Sherman 2002). Cells were transformed by the lithium acetate method (Chen et al. 1992). The CUP1 promoter was induced with $0.1 \mathrm{mM} \mathrm{CuSO}_{4}$.

\section{Plasmids and plasmid construction}

The plasmids used are: YIpHA-RSP5 (this work), YIpHA-rsp5-13 (this work), YCp33HA-RSP5 (Gajewska et al. 2001), YEp-NPI1/ RSP5 (from B. Andre, Universite Libre de Bruxelles, Belgium), 
YCp-TEF2/JWB2828 (from T. Kinzy, University of Medicine and Dentistry of New Jersey, Piscataway), YEp96 (Ecker et al. 1987), pACTQ, pAC1789, pACTy, pACTMV (Stahl et al. 1995), pACTAA, and pACTGA (Bidou et al. 2000). The YIpHA-RSP5 plasmid used for integration into the MHY501 strain was constructed by cloning the EcoRI-SphI DNA fragment obtained by digestion of YCp33HA-RSP5 (Gajewska et al. 2001) into vector YIplac211 (Gietz and Sugino 1988). The YIpHA-rsp5-13 was obtained by substituting the AgeI-MunI fragment in YIpHA-RSP5 with the DNA fragment, synthesized in a PCR reaction using specific primers and genomic DNA from TZ23 rsp5-13 strain as the template (Żołądek et al. 1997) and digested by AgeI and MunI enzymes.

DNA restriction and electrophoresis were carried out according to standard procedures (Sambrook and Russell 2001). PCR reactions and sequencing were performed on double-stranded DNA, and sequencing was carrying out using an automatic sequencer $\mathrm{ABI} 310$ Perkin Elmer in the DNA Sequencing and Oligonucleotide Synthesis Laboratory, Institute of Biochemistry and Biophysics, Polish Academy of Sciences. Oligonucleotides used were synthesized at the above laboratory and their sequences are available upon request.

\section{Readthrough frequency analysis}

The reporter plasmids, pACTy (+1 frameshifting), pAC1789 (-1 frameshifting), pACTMV (UAG readthrough), pACTGA (UGA readthrough), pACTAA (UAA readthrough), and pACTQ (in frame control), were transformed into yeast strains (Stahl et al. 1995; Bidou et al. 2000). At least two transformants, cultivated at $30^{\circ} \mathrm{C}$ in $\mathrm{SD}+\mathrm{CSM}$, were assayed in a given experiment. Cells were harvested at an $\mathrm{OD}_{600}$ of $1.5-3$ and disrupted using glass beads. Luciferase and $\beta$-galactosidase activities were assayed as described (Stahl et al. 1995). The recoding efficiency, expressed in percentages, was calculated by dividing the luciferase/ $\beta$-galactosidase ratio obtained using each test plasmid by the same ratio obtained with the in-frame control plasmid (Bidou et al. 2000). Results are the mean of at least five independent experiments and were tested by a Mann-Whitney $u$-test (Lowry 2000).

\section{Fluorescence in situ hybridization}

For FISH, published procedures and oligonucleotides were employed (Sarkar and Hopper 1998; Sarkar et al. 1999; Feng and Hopper, 2002). Strains were grown at $23^{\circ} \mathrm{C}$ to $\log$ phase in YPD or selective media SC-ura or SD + casamino acids and subsequently shifted to $37^{\circ} \mathrm{C}$ for the $3.5 \mathrm{~h}$. For the TEF2 suppression experiment strains were grown at $23^{\circ} \mathrm{C}$ to $\log$ phase and subsequently shifted to $33.5^{\circ} \mathrm{C}$ for the $3.5 \mathrm{~h}$. Digoxigenin-labeled oligonucleotide probes specific for intronless tRNA $\left(\mathrm{tRNA}^{\mathrm{Met}}\right)$ or recognizing intron-containing pre-tRNA and processed tRNA (tRNA ${ }^{\text {Tyr }}$ ) were used. Fluorescence images were observed by using a Nikon Microphot-FX microscope, captured with a SenSys chargecoupled device camera (Photometrics) with QED software (QED Imaging) and assembled with Adobe Photoshop 5.0.

\section{In vivo $\left[{ }^{35} S\right]$-methionine incorporation}

An overnight YPD culture at $\mathrm{OD}_{600} 0.8-1.1$ was spun down, suspended in $\mathrm{P}$ buffer $(40 \mathrm{mM}$ potassium phosphate buffer at $\mathrm{pH} 7.4,0.45 \%$ glucose, $0.0077 \%$ SD + CSM-methionine), and incubated with shaking at $30^{\circ} \mathrm{C}$ for $30 \mathrm{~min}$. Forty microcuries of $\left[{ }^{35} \mathrm{~S}\right]$-methionine were added and samples were withdrawn at the time points indicated. Incorporation of labeled methionine was stopped by adding $1 / 10$ volume of $0.2 \mathrm{M}$ unlabeled methionine. Cells were filtered under vacuum onto GF/A glass microfiber filters (Whatman), washed with 10\% TCA, 95\% ethanol, and acetone, and dried. Radioactivity was measured in a scintillation counter (Ciechanover et al. 1984, with modifications).

\section{ACKNOWLEDGMENTS}

We thank T. Kinzy for YCp-TEF2/JWB2828, B. Andre for YEpNPI1/RSP5, and B. Gajewska for construction of YIpHA-rsp5-13 plasmid. We thank S. Herman-Le Denmat for kind help in polysome profile analysis. We also thank all members of T. Żołądek, J. P.Rousset, M. Boguta, and A.K. Hopper's laboratories for help and numerous scientific interactions. This work was supported by the State Committee for Scientific Research of Poland grant 3P04B01624 to T.Z.; travel grant from PNCMB UNESCO/PAS and thesis grant from Franco-Polonais Réseau de FormationRecherches (Ministère de l'Education Nationale, de l'Enseignement Supérieur et de la Recherche) to M.K.; Association pour la Recherche sur le Cancer (contract 4699) and Association Française contre les Myopathies for J.-P.R.; and a grant from the National Institutes of Health (GM27930) to A.K.H.

Received June 3, 2005; accepted August 12, 2005.

\section{REFERENCES}

Abraham, A.K. and Pihl, A. 1983. Effect of protein synthesis inhibitors on the fidelity of translation in eukaryotic systems. Biochim. Biophys. Acta 741: 197-203.

Beck, T., Schmidt, A., and Hall, M.N. 1999. Starvation induces vacuolar targeting and degradation of the tryptophan permease in yeast. J. Cell Biol. 146: 1227-1238.

Beier, H. and Grimm, M. 2001. Misreading of termination codons in eukaryotes by natural nonsense suppressor tRNAs. Nucleic Acids Res. 29: 4767-4782.

Bidou, L., Stahl, G., Hatin, I., Namy, O., Rousset, J.P., and Farabaugh, P.J. 2000. Nonsense-mediated decay mutants do not affect programmed -1 frameshifting. RNA 6: 952-961.

Carr-Schmid, A., Durko, N., Cavallius, J., Merrick, W.C., and Kinzy, T.G. 1999. Mutations in a GTP-binding motif of eukaryotic elongation factor $1 \mathrm{~A}$ reduce both translational fidelity and the requirement for nucleotide exchange. J. Biol. Chem. 274: 3029730302.

Chen, D.C., Yang, B.C., and Kuo, T.T. 1992. One-step transformation of yeast in stationary phase. Curr. Genet. 21: 83-84.

Chen, P., Johnson, P., Sommer, T., Jentsch, S., and Hochstrasser, M. 1993. Multiple ubiquitin-conjugating enzymes participate in the in vivo degradation of the yeast MAT $\alpha 2$ repressor. Cell 74: 357-369.

Chernova, T.A., Allen, K.D., Wesoloski, L.M., Shanks, J.R., Chernoff, Y.O., and Wilkinson, K.D. 2003. Pleiotropic effects of Ubp6 loss on drug sensitivities and yeast prion are due to depletion of the free ubiquitin pool. J. Biol. Chem. 278: 52102-52115.

Ciechanover, A., Finley, D., and Varshavsky, A. 1984. Ubiquitin dependence of selective protein degradation demonstrated in the mammalian cell cycle mutant ts85. Cell 37: 57-66.

Crespo, J.L., Helliwell, S.B., Wiederkehr, C., Demougin, P., Fowler, B., Primig, M., and Hall, M.N. 2004. NPR1 kinase and RSP5-BUL1/2 ubiquitin ligase control GLN3-dependent transcription in Saccharomyces cerevisiae. J. Biol. Chem. 279: 37512-37517. 
de la Fuente, N., Maldonado, A.M., and Portillo, F. 1997. Glucose activation of the yeast plasma membrane $\mathrm{H}^{+}$-ATPase requires the ubiquitin-proteasome proteolytic pathway. FEBS Lett. 411: 308312.

Dihanich, M.E., Najarian, D., Clark, R., Gillman, E.C., Martin, N.C., and Hopper, A.K. 1987. Isolation and characterization of MOD5, a gene required for isopentenylation of cytoplasmic and mitochondrial tRNAs of Saccharomyces cerevisiae. Mol. Cell. Biol. 7: 177-184.

Dinman, J.D. and Kinzy, T.G. 1997. Translational misreading: Mutations in translation elongation factor $1 \alpha$ differentially affect programmed ribosomal frameshifting and drug sensitivity. RNA 3: 870-881.

Dunn, R. and Hicke, L. 2001a. Domains of the Rsp5 ubiquitin-protein ligase required for receptor-mediated and fluid-phase endocytosis. Mol. Biol. Cell 12: 421-435.

- 2001b. Multiple roles for Rsp5p-dependent ubiquitination at the internalization step of endocytosis. J. Biol. Chem. 276: 2597425981.

Ecker, D.J., Khan, M.I., Marsh, J., Butt, T.R., and Crooke, S.T. 1987. Chemical synthesis and expression of a cassette adapted ubiquitin gene. J. Biol. Chem. 262: 3524-3527.

Feng, W. and Hopper, A.K. 2002. A Loslp-independent pathway for nuclear export of intronless tRNAs in Saccharomyces cerevisiae. Proc. Natl. Acad. Sci. 99: 5412-5417.

Finley, D., Ozkaynak, E., and Varshavsky, A. 1987. The yeast polyubiquitin gene is essential for resistance to high temperatures, starvation, and other stresses. Cell 48: 1035-1046.

Finley, D., Bartel, B., and Varshavsky, A. 1989. The tails of ubiquitin precursors are ribosomal proteins whose fusion to ubiquitin facilitates ribosome biogenesis. Nature 338: 394-401.

Gajewska, B., Kamińska, J., Jesionowska, A., Martin, N.C., Hopper, A.K., and Żołądek, T. 2001. WW domains of Rsp5p define different functions: Determination of roles in fluid phase and uracil permease endocytosis in Saccharomyces cerevisiae. Genetics 157: 91-101.

Galan, J.M., Moreau, V., Andre, B., Volland, C., and HaguenauerTsapis, R. 1996. Ubiquitination mediated by the Npilp/Rsp5p ubiquitin-protein ligase is required for endocytosis of the yeast uracil permease. J. Biol. Chem. 271: 10946-10952.

Gietz, R.D. and Sugino, A. 1988. New yeast-Escherichia coli shuttle vectors constructed with in vitro mutagenized yeast genes lacking six-base pair restriction sites. Gene 74: 527-534.

Grosshans, H., Hurt, E., and Simos, G. 2000a. An aminoacylationdependent nuclear tRNA export pathway in yeast. Genes \& Dev. 14: 830-840.

Grosshans, H., Simos, G., and Hurt, E. 2000b. Review: Transport of tRNA out of the nucleus-Direct channeling to the ribosome? J. Struct. Biol. 129: 288-294.

Gwizdek, C., Hobeika, M., Kus, B., Ossareh-Nazari, B., Dargemont, C., and Rodriguez, M.S. 2005. The mRNA nuclear export factor Hpr1 is regulated by Rsp5-mediated ubiquitylation. J. Biol. Chem. 280: 13401-13405.

Hanna, J., Leggett, D.S., and Finley, D. 2003. Ubiquitin depletion as a key mediator of toxicity by translational inhibitors. Mol. Cell. Biol. 23: 9251-9261.

Harvey, K.F. and Kumar, S. 1999. Nedd4-like proteins: An emerging family of ubiquitin-protein ligases implicated in diverse cellular functions. Trends Cell Biol. 9: 166-169.

Hein, C., Springael, J.Y., Volland, C., Haguenauer-Tsapis, R., and Andre, B. 1995. NPI1, an essential yeast gene involved in induced degradation of Gap1 and Fur4 permeases, encodes the Rsp5 ubiquitin-protein ligase. Mol. Microbiol. 18: 77-87.

Helliwell, S.B., Losko, S., and Kaiser, C.A. 2001. Components of a ubiquitin ligase complex specify polyubiquitination and intracellular trafficking of the general amino acid permease. J. Cell Biol. 153: 649-662.

Hellmuth, K., Lau, D.M., Bischoff, F.R., Kunzler, M., Hurt, E., and Simos, G. 1998. Yeast Los1p has properties of an exportin-like nucleocytoplasmic transport factor for tRNA. Mol. Cell. Biol. 18: 6374-6386.
Hershko, A. and Ciechanover, A. 1998. The ubiquitin system. Annu. Rev. Biochem. 67: 425-479.

Hicke, L. 2001. Protein regulation by monoubiquitin. Nat. Rev. Mol. Cell. Biol. 2: 195-201.

Hitchcock, A.L., Auld, K., Gygi, S.P., and Silver, P.A. 2003. A subset of membrane-associated proteins is ubiquitinated in response to mutations in the endoplasmic reticulum degradation machinery. Proc. Natl. Acad. Sci. 100: 12735-12740.

Hochstrasser, M. 1996. Ubiquitin-dependent protein degradation. Annu. Rev. Genet. 30: 405-439.

Hoppe, T., Matuschewski, K., Rape, M., Schlenker, S., Ulrich, H.D., and Jentsch, S. 2000. Activation of a membrane-bound transcription factor by regulated ubiquitin/proteasome-dependent processing. Cell 102: 577-586.

Hopper, A.K. and Phizicky, E.M. 2003. tRNA transfers to the limelight. Genes \& Dev. 17: 162-180.

Hopper, A.K., Schultz, L.D., and Shapiro, R.A. 1980. Processing of intervening sequences: A new yeast mutant which fails to excise intervening sequences from precursor tRNAs. Cell 19: 741-751.

Huibregtse, J.M., Scheffner, M., Beaudenon, S., and Howley, P.M. 1995. A family of proteins structurally and functionally related to the E6-AP ubiquitin-protein ligase. Proc. Natl. Acad. Sci. 92: 5249.

Hurt, D.J., Wang, S.S., Lin, Y.H., and Hopper, A.K. 1987. Cloning and characterization of LOS1, a Saccharomyces cerevisiae gene that affects tRNA splicing. Mol. Cell. Biol. 7: 1208-1216.

Kabir, M.A., Kamińska, J., Segel, G.B., Bethlendy, G., Lin, P., Della, S.F., Blegen, C., Swiderek, K.M., Żołądek, T., Arndt, K.T., et al. 2005. Physiological effects of unassembled chaperonin Cct subunits in the yeast Saccharomyces cerevisiae. Yeast 22: 219-239.

Kamińska, J., Tobiasz, A., Gniewosz, M., and Żołądek, T. 2000. The growth of mdp1/rsp5 mutants of Saccharomyces cerevisiae is affected by mutations in the ATP-binding domain of the plasma membrane H+-ATPase. Gene 242: 133-140.

Kamińska, J., Gajewska, B., Hopper, A.K., and Żołądek, T. 2002. Rsp5p, a new link between the actin cytoskeleton and endocytosis in the yeast Saccharomyces cerevisiae. Mol. Cell. Biol. 22: 6946-6948.

Kandl, K.A., Munshi, R., Ortiz, P.A., Andersen, G.R., Kinzy, T.G., and Adams, A.E. 2002. Identification of a role for actin in translational fidelity in yeast. Mol. Genet. Genomics 268: 10-18.

Katzmann, D.J., Sarkar, S., Chu, T., Audhya, A., and Emr, S.D. 2004. Multivesicular body sorting: Ubiquitin ligase Rsp5 is required for the modification and sorting of carboxypeptidase S. Mol. Biol. Cell 15: 468-480.

Keeven, J., Ko, D., Shallom, J., Uccelini, B., and Golin, J. 2002. PDR2 gain-of-function mutations eliminate the need for Pdr1 and require the UBP6 product for resistance to translational inhibitors. Curr. Genet. 41: 11-19.

Krsmanovic, T. and Kolling, R. 2004. The HECT E3 ubiquitin ligase Rsp5 is important for ubiquitin homeostasis in yeast. FEBS Lett. 577: 215-219.

Kurland, C.G. 1992. Translational accuracy and the fitness of bacteria. Annu. Rev. Genet. 26: 29-50.

Kwapisz, M., Smagowicz, W.J., Oficjalska, D., Hatin, I., Rousset, J.P., Żołądek, T., and Boguta, M. 2002. Up-regulation of tRNA biosynthesis affects translational readthrough in maf1- $\Delta$ mutant of Saccharomyces cerevisiae. Curr. Genet. 42: 147-152.

Lecointe, F., Namy, O., Hatin, I., Simos, G., Rousset, J.P., and Grosjean, H. 2002. Lack of pseudouridine 38/39 in the anticodon arm of yeast cytoplasmic tRNA decreases in vivo recoding efficiency. J. Biol. Chem. 277: 30445-30453.

Lindsten, K., de Vrij, F.M., Verhoef, L.G., Fischer, D.F., van Leeuwen, F.W., Hol, E.M., Masucci, M.G., and Dantuma, N.P. 2002. Mutant ubiquitin found in neurodegenerative disorders is a ubiquitin fusion degradation substrate that blocks proteasomal degradation. J. Cell Biol. 157: 417-427.

Lowry, R. 2000. $t$-Test for the significance of the difference between the means of two independent samples: The Mann-Whitney test. In Concepts and applications of inferential statistics, http://faculty. vassar.edu/lowry/webtext.html. Vassar College, Poughkeepsie, NY. 
Masurekar, M., Palmer, E., Ono, B.I., Wilhelm, J.M., and Sherman, F. 1981. Misreading of the ribosomal suppressor SUP46 due to an altered $40 \mathrm{~S}$ subunit in yeast. J. Mol. Biol. 147: 381-390.

Moazed, D. and Noller, H.F. 1987. Interaction of antibiotics with functional sites in 16S ribosomal RNA. Nature 327: 389-394.

Morvan, J., Froissard, M., Haguenauer-Tsapis, R., and Urban-Grimal, D. 2004. The ubiquitin ligase Rsp5p is required for modification and sorting of membrane proteins into multivesicular bodies. Traffic 5: 383-392.

Neumann, S., Petfalski, E., Brugger, B., Grosshans, H., Wieland, F., Tollervey, D., and Hurt, E. 2003. Formation and nuclear export of tRNA, rRNA and mRNA is regulated by the ubiquitin ligase Rsp5p. EMBO Rep. 4: 1156-1162.

Ogle, J.M., Brodersen, D.E., Clemons Jr., W.M., Tarry, M.J., Carter, A.P., and Ramakrishnan, V. 2001. Recognition of cognate transfer RNA by the 30S ribosomal subunit. Science 292: 897-902.

Ozkaynak, E., Finley, D., and Varshavsky, A. 1984. The yeast ubiquitin gene: Head-to-tail repeats encoding a polyubiquitin precursor protein. Nature 312: 663-666.

Peng, J., Schwartz, D., Elias, J.E., Thoreen, C.C., Cheng, D., Marsischky, G., Roelofs, J., Finley, D., and Gygi, S.P. 2003. A proteomics approach to understanding protein ubiquitination. Nat. Biotechnol. 21: 921-926.

Rodriguez, M.S., Gwizdek, C., Haguenauer-Tsapis, R., and Dargemont, C. 2003. The HECT ubiquitin ligase Rsp5p is required for proper nuclear export of mRNA in Saccharomyces cerevisiae. Traffic 4: $566-575$.

Rodriguez-Fonseca, C., Amils, R., and Garrett, R.A. 1995. Fine structure of the peptidyl transferase centre on 23 S-like rRNAs deduced from chemical probing of antibiotic-ribosome complexes. J. Mol. Biol. 247: 224-235.

Sambrook, J. and Russell, D.W. 2001. Molecular cloning. A laboratory manual. Cold Spring Harbor Laboratory Press, Cold Spring Harbor, NY.

Sarkar, S. and Hopper, A.K. 1998. tRNA nuclear export in Saccharomyces cerevisiae: In situ hybridization analysis. Mol. Biol. Cell 9: 3041-3055.

Sarkar, S., Azad, A.K., and Hopper, A.K. 1999. Nuclear tRNA aminoacylation and its role in nuclear export of endogenous tRNAs in Saccharomyces cerevisiae. Proc. Natl. Acad. Sci. 96: 14366-14371.

Schwartz, D.C. and Hochstrasser, M. 2003. A superfamily of protein tags: Ubiquitin, SUMO and related modifiers. Trends Biochem. Sci. 28: $321-328$.

Shaheen, H. and Hopper, A.K. 2005. Retrograde movement of tRNAs from the cytoplasm to the nucleus in S. cerevisiae. Proc. Natl. Acad. Sci. 102: 11290-11295.

Shcherbik, N., Żołądek, T., Nickels, J.T., and Haines, D.S. 2003. Rsp5p is required for ER bound Mga2p120 polyubiquitination and release of the processed/tethered transactivator Mga2p90. Curr. Biol. 13: 1227-1233.
Shcherbik, N., Kee, Y., Lyon, N., Huibregtse, J.M., and Haines, D.S. 2004. A single PXY motif located within the carboxyl terminus of Spt23p and Mga2p mediates a physical and functional interaction with ubiquitin ligase Rsp5p. J. Biol. Chem. 51: 53892-53898.

Sherman, F. 2002. Getting started with yeast. Methods Enzymol. 350: $3-41$.

Song, J.M., Picologlou, S., Grant, C.M., Firoozan, M., Tuite, M.F., and Liebman, S. 1989. Elongation factor EF-1 $\alpha$ gene dosage alters translational fidelity in Saccharomyces cerevisiae. Mol. Cell. Biol. 9: 4571-4575.

Spence, J., Gali, R.R., Dittmar, G., Sherman, F., Karin, M., and Finley, D. 2000. Cell cycle-regulated modification of the ribosome by a variant multiubiquitin chain. Cell 102: 67-76.

Springael, J.Y., De Craene, J.O., and Andre, B. 1999. The yeast Npil/ Rsp5 ubiquitin ligase lacking its N-terminal C2 domain is competent for ubiquitination but not for subsequent endocytosis of the gap1 permease. Biochem. Biophys. Res. Commun. 257: 561-566.

Stahl, G., Bidou, L., Rousset, J.P., and Cassan, M. 1995. Versatile vectors to study recoding: Conservation of rules between yeast and mammalian cells. Nucleic Acids Res. 23: 1557-1560.

Stamenova, S.D., Dunn, R., Adler, A.S., and Hicke, L. 2004. The Rsp5 ubiquitin ligase binds to and ubiquitinates members of the yeast CIN85-endophilin complex, Sla1-Rvs167. J. Biol. Chem. 279: 16017-16025.

Takano, A., Endo, T., and Yoshihisa, T. 2005. tRNA actively shuttles between the nucleus and cytosol in yeast. Science 309: $140-142$.

Valente, L. and Kinzy, T.G. 2003. Yeast as a sensor of factors affecting the accuracy of protein synthesis. Cell. Mol. Life Sci. 60: 2115-2130.

Vicens, Q. and Westhof, E. 2001. Crystal structure of paromomycin docked into the eubacterial ribosomal decoding A site. Structure (Camb.) 9: 647-658.

Wang, G., Yang, J., and Huibregtse, J.M. 1999. Functional domains of the Rsp5 ubiquitin-protein ligase. Mol. Cell. Biol. 19: 342-352.

Weissman, A.M. 2001. Themes and variations on ubiquitylation. Nat. Rev. Mol. Cell. Biol. 2: 169-178.

Wolfe, D., Reiner, T., Keeley, J.L., Pizzini, M., and Keil, R.L. 1999. Ubiquitin metabolism affects cellular response to volatile anesthetics in yeast. Mol. Cell. Biol. 19: 8254-8262.

Żołądek, T., Vaduva, G., Hunter, L.A., Boguta, M., Go, B.D., Martin, N.C., and Hopper, A.K. 1995. Mutations altering the mitochondrial-cytoplasmic distribution of Mod5p implicate the actin cytoskeleton and mRNA $3^{\prime}$ ends and/or protein synthesis in mitochondrial delivery. Mol. Cell. Biol. 15: 68846894.

Żołądek, T., Tobiasz, A., Vaduva, G., Boguta, M., Martin, N.C., and Hopper, A.K. 1997. MDP1, a Saccharomyces cerevisiae gene involved in mitochondrial/cytoplasmic protein distribution, is identical to the ubiquitin-protein ligase gene RSP5. Genetics 145: 595-603. 
RNA 11: 1710-1718 (2005)

\section{Rsp5 ubiquitin ligase modulates translation accuracy in yeast Saccharomyces cerevisiae}

\section{MARTA KWAPISZ, PIOTR CHOŁBIŃSKI, ANITA K. HOPPER, JEAN-PIERRE ROUSSET, and TERESA ŻOŁĄDEK}

The strain that was used in this study designated as MK5 contains mutation rsp5-19, resulting in P418L substitution in WW domain of Rsp5, and not of rsp5-13 mutation resulting in substitution G707D in Hect domain of Rsp5, as published. The name of this strain should be MK15. All other published data in this paper are correct. The authors apologize any confusion these errors may have caused. The errors do not change the conclusions of the paper.

Specifically, the changes in the paper are listed below:

- In Results, page 1711, the last sentence in the first column should begin: "The rsp5-19 mutation results in P418L substitution in WW3 domain of Rsp5 ..."

- In Materials and Methods, page 1715, Materials and Methods, the first sentence beneath the first subheading after the citation (Chen et al. 1993) should read: "MK15 (MHY501 but rsp5-19; this work)." In addition, the name of the mutation should be $r s p-19$ and the name of the strain should be MK15 throughout the publication (not $r s p 5-13$ and MK5, as published).

- In Materials and Methods, page 1715, beneath the subhead Plasmids and plasmid construction, the name of the plasmid at the end of the third line from the bottom of the page should be YIpHA-rsp5-19 (not YIpHA-rsp5-13). This is also the case on page 1716 , line 8 .

- In Materials and Methods, page 1716, line 11, the name of strain should be TZ29 rsp5-19 (not TZ23 rsp5-13). 

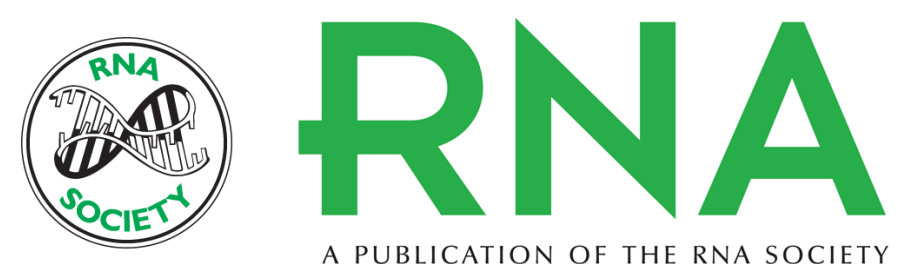

A PUBLICATION OF THE RNA SOCIETY

\section{Rsp5 ubiquitin ligase modulates translation accuracy in yeast Saccharomyces cerevisiae}

MARTA KWAPISZ, PIOTR CHOLBINSKI, ANITA K. HOPPER, et al.

RNA 2005 11: 1710-1718

Related Content

References

License

Email Alerting Service
ERRATUM

RNA March , 2006 12: 530

This article cites 77 articles, 36 of which can be accessed free at: http://rnajournal.cshlp.org/content/11/11/1710.full.html\#ref-list-1

Articles cited in:

http://rnajournal.cshlp.org/content/11/11/1710.full.html\#related-urls

Receive free email alerts when new articles cite this article - sign up in the box at the top right corner of the article or click here. 